\title{
Evaluation of Endoscopic Sinus Surgery Combined with Dexamethasone in the Treatment of Chronic Rhinosinusitis
}

\author{
Y. Q. LI*, L. L. SHENG, Q. DING AND H. X. SHAO \\ Department of E. N. T., The First People's Hospital of Chuzhou City, No. 4, Zhongyou Lane, Gulou Street, Chuzhou, Anhui \\ 239000, China
}

Li et al:: Endoscopic Sinus Surgery and Dexamethasone for Chronic Rhinosinusitis

\begin{abstract}
The purpose of this study is to explore the efficacy of endoscopic sinus surgery combined with dexamethasone for treating chronic rhinosinusitis. A total of 123 chronic rhinosinusitis patients diagnosed by magnetic resonance imaging and cochlear implant in the otolaryngology department of the First People's Hospital of Chuzhou City from January 2018 to January 2019 were selected. They were randomly divided into the control group $(n=61)$ and the experimental group $(n=62)$. After endoscopic sinus surgery, the control group used only normal saline for nasal cleaning, while the experimental group added dexamethasone nasal spray for regular treatment. After outpatient follow-up every $3 \mathrm{mo}$, the sinonasal outcome test-20 scores, Lund-Kennedy scores, olfactory tests, and therapeutic effects were compared between the two groups. Before treatment, compared to the control group, there was no significant difference in the sinonasal outcome test-20 scores $(38.78 \pm 5.42$ vs $39.08 \pm 5.73)$ and Lund-Kennedy scores $(17.35 \pm 3.21$ vs $17.40 \pm 3.35)$ of the experimental group. After treatment, during the follow-up period of 3,6 , and $12 \mathrm{mo}$, the sinonasal outcome test-20 scores were $(9.38 \pm 2.10$ vs $2.10 \pm 0.45)$ and the Lund-Kennedy scores were $(7.45 \pm 1.65$ vs $2.23 \pm 0.73)$ between the control and the experimental groups, which were significantly different between the two groups $(P<0.05)$. The olfactory test scores of the experimental group was significantly higher $(\mathbf{P}<0.05)$. After treatment for $12 \mathrm{mo}$, the cure rate of the experimental group was $95.16 \%$, which was significantly
\end{abstract}


higher than that of the control group cure rate of $90.16 \%$. In terms of the recurrence rate after treatment, there was a statistically significant difference between the two groups in the follow-up period of 3 mo $(22.95$ vs $12.90 \%)$ and 6 mo (14.75 vs 6.45\%). However, there was no significant difference in the followup period of $12 \mathrm{mo}$ (1.45 vs 0). Endoscopic sinus surgery combined with dexamethasone could ameliorate the clinical symptoms of chronic rhinosinusitis patients, improve their quality of life. It also has a better long-term effect, which is worth to consider.

Key words: Chronic rhinosinusitis; endoscopic sinus surgery; dexamethasone; recurrence rate

Chronic rhinosinusitis (CRS) was a kind of chronic inflammatory disease of the nose or paranasal sinus mucosa, with clinical symptoms such as nasal obstruction, purulent discharge and olfactory dysfunction, which has not been relieved for a long time (generally $3 \mathrm{mo}$ ). It usually occurs in people with low immunity. Many other complications such as respiratory tract infections, intracranial infections and decreased vision were often seen in the severe patients, seriously lowering the quality of life ${ }^{[1]}$. Endoscopic sinus surgery (ESS), a kind of common surgery of paranasal sinus, has been proved to be effective in the treatment of chronic rhino-sinusitis. It may cause potentially avoidable complications of mucositis, such as bone stenosis and adhesion $^{[2-3]}$. Therefore, the mucosal inflammation was an important goal of early nursing after ESS. So, the main clinical treatment for CRS at present is ESS and the administration of a glucocorticoid. However, there is a controversy about the combination of the two treatments and the administration methods ${ }^{[3-4]}$. Therefore, the purpose of this study is to explore the efficacy of ESS combined with dexamethasone in the treatment of CRS. A total of 168 CRS patients diagnosed by magnetic resonance imaging (MRI) and cochlear implant (CI) in the otolaryngology department of the First People's Hospital of Chuzhou City from January 2018 to January 2019 were selected. Exclusion criteria were, those patients with severe cardiovascular disease or mental illness and a family history of mental illness. Patients with surgical contraindications. Patients younger than $18 \mathrm{y}$. Patients with systemic inflammatory disease. Finally, 123 cases were included in this study and randomly divided into the experimental group $(n=62)$ and the control group $(n=61)$ by the computergenerated random number. In the control group, there were 37 males and 25 females (21-56 y old, mean age $36.34 \pm 7.88 \mathrm{y}$; the course of the disease was from 1 to 9 y (mean $4.78 \pm 2.13 \mathrm{y}$ ). In the experimental group, there were 35 males and 26 females (19-58 y old, mean age $35.31 \pm 6.40 \mathrm{y}$ and the course of the disease was from 2 to $8 \mathrm{y}$ (mean $4.88 \pm 2.42 \mathrm{y}$ ). There was no significant difference between the two groups general clinical data. All patients were treated with ESS after oral intubation and general anesthesia. According to the focus location of the patients, the patients were divided into 3 different categories, anterior and posterior ethmoid sinuses, frontal sinuses, and open maxillary sinuses. The focus and nasal polyps were removed and cleaned, and the abnormal nasal cavity structure was corrected at the same time. After ESS, the blood stopping gauze and hemostatic sponge were filled in the nasal cavity and tissues removed were examined for pathological findings. Within 2-3 d after the operation, the hemostatic sponges of all patients were removed and the residual blood and secretion were cleaned. On the $\mathrm{d} 5$, all patients were given a nasal cleaner and saline was once a day for 6 mo. In addition, the patients in the experimental group were given dexamethasone nasal spray on each side of the nasal cavity twice a day for $6 \mathrm{mo}$. Before leaving the hospital, the patients were instructed and taught the correct nursing way to wash nasal cavity to get an effect of continuing treatment. At the same time, the patients had an outpatient reexamination every 3 mo, up to $1 \mathrm{y}$. The reexamination mainly included the disease recovery examination and treatment effect evaluation. Subjective evaluation index such as the nasal sinus outcome test-20 (SNOT-20) scale was used in this study ${ }^{[5]}$. It covered the self-perception evaluation of the psychological state, nasal symptoms, and physical functions of the patients in the treatment. Corresponding scores were obtained according to the patients' subjective feelings. The lower the score, the better the treatment. Lund-Kennedy scale ${ }^{[6]}$ was used for the objective evaluation of the treatment effects in the two groups, which mainly based on the postoperative recovery of patients' nasal cavity, including whether there were polyp, edema, scar, adhesion. The lower the scores, the better the recovery of patients. Olfactory fluids were used for the detection of the olfactory sensation $^{[7]}$. Ten olfactory test fluids with different 
concentrations were prepared according to the olfactory elements with a certain concentration gradient and the score was from 1 to 10 based on the concentration from high to low. The olfactory evaluation index was the average threshold of the two groups after olfactory tests. The criteria were normal (average threshold $>3$ points), mild disorder (average threshold between 2.1 -3 points), moderate disorder (between 1.1-2 points), and severe disorder (average threshold less than 1 point). Olfactory monitoring tests were evaluated before the treatment and during the last follow-up period.

Relapse was evaluated by checking the nasal cavity of the patients while cleaning when they underwent the reexamination. The cure rate and recurrence rate of the patients were counted during each reexamination. The cure standards were, no clinical symptom of sinusitis; the nasal sinus ostia were unobstructed; the mucous epithelium recovered well; no edema and mucinous secretion were found in the nasal cavity. Recurrence criteria were, there were extensive congestion and edema in the nasal cavity, with polyp and connective tissue hyperplasia, and sinus stenosis and whether the clinical symptoms of the patients were not improved or became worse. SPSS20.0 software was used for statistical calculation, with a T-test and Chi-square test. $P<0.05$ means the difference was statistically significant. Before treatment, there was no significant difference in SNOT-20 scores between the experimental group and the control group. After treatment, in the follow-up period of 3, 6, and $12 \mathrm{mo}$, compared to the control group, the SNOT-20 scores of the experimental group decreased significantly $(P<0.05$, Table 1$)$. Before treatment, there was no significant difference in LundKennedy scores between the 2 groups but after treatment, during the follow-up period of 3,6 , and $12 \mathrm{mo}$, the Lund-Kennedy scores of the experimental group were significantly lower than that of the control group $(P<0.05$, Table 2$)$. Before treatment, there was no significant difference $(P>0.05)$ in the olfactory test scores between the 2 groups. After treatment, the olfactory test scores of the experimental group were significantly higher than that of the control group $(P<0.05)$. And after treatment, the olfactory functions of all patients in each group were improved significantly (Table 3). These results showed that after treatment, compared with the control group, in the follow-up period of 3,6 , and $12 \mathrm{mo}$, the cure rate of patients in the experimental group was significantly higher, and the difference between the two groups was statistically significant $(P<0.05)$ (Table 4$)$. In terms of the recurrence rate, after treatment, there was a statistically significant difference $(P>0.05)$ between the two groups in the follow-up period of 3 mo and 6 mo (Table 5). However, there was no significant difference in the follow-up

TABLE 1: COMPARISON OF THE SNOT-20 SCORES BEFORE AND AFTER TREATMENT

\begin{tabular}{|c|c|c|c|c|c|}
\hline \multirow{2}{*}{ Group } & \multirow{2}{*}{ Cases } & \multicolumn{4}{|c|}{ SNOT-20 score } \\
\hline & & Before treatment & $3 \mathrm{mo}$ & $6 \mathrm{mo}$ & $12 \mathrm{mo}$ \\
\hline Control & 61 & $38.78 \pm 5.42$ & $22.55 \pm 4.21$ & $16.03 \pm 3.85$ & $9.38 \pm 2.10$ \\
\hline Experimental & 62 & $39.08 \pm 5.73$ & $9.52 \pm 3.37$ & $6.67 \pm 2.34$ & $2.10 \pm 0.45$ \\
\hline $\mathrm{t}$ & & 0.0863 & 11.524 & 9.336 & 16.430 \\
\hline$P$ & & 0.924 & $<0.05$ & $<0.05$ & $<0.05$ \\
\hline
\end{tabular}

TABLE 2: COMPARISON OF LUND-KENNEDY SCORES BEFORE AND AFTER TREATMENT

\begin{tabular}{|c|c|c|c|c|c|}
\hline \multirow{2}{*}{ Group } & \multirow{2}{*}{ Cases } & \multicolumn{4}{|c|}{ Lund-Kennedy score } \\
\hline & & Before treatment & $3 \mathrm{mo}$ & $6 \mathrm{mo}$ & $12 \mathrm{mo}$ \\
\hline Control & 61 & $17.35 \pm 3.21$ & $14.71 \pm 2.25$ & $11.55 \pm 1.96$ & $7.45 \pm 1.65$ \\
\hline Experimental & 62 & $17.40 \pm 3.35$ & $10.02 \pm 2.05$ & $6.21 \pm 1.67$ & $2.23 \pm 0.73$ \\
\hline $\mathrm{t}$ & & 0.0660 & 7.005 & 10.550 & 12.850 \\
\hline$P$ & & 0.935 & $<0.05$ & $<0.05$ & $<0.05$ \\
\hline
\end{tabular}

TABLE 3: COMPARISON OF THE OLFACTORY TESTS BEFORE AND AFTER TREATMENT

\begin{tabular}{lcccc}
\hline \multirow{2}{*}{ Group } & \multicolumn{2}{c}{ Experimental group } & \multicolumn{2}{c}{ Control group } \\
\cline { 2 - 5 } & Before treatment & 12 mo & Before treatment & 12 mo \\
\hline Normal $\mathrm{n}(\%)$ & $3(4.84 \%)$ & $58(93.55 \%)^{\mathrm{a} *}$ & $4(6.56 \%)$ & $45(73.77 \%)^{\mathrm{b}}$ \\
Mild disorder $\mathrm{n}(\%)$ & $17(27.42 \%)$ & $4(6.45 \%)^{\mathrm{a}}$ & $18(29.51 \%)$ & $15(24.59 \%)^{\mathrm{b}}$ \\
Moderate disorder $\mathrm{n}(\%)$ & $36(58.06 \%)$ & $0(0)^{\mathrm{a}}$ & $34(55.74 \%)$ & $1(1.64 \%)^{\mathrm{b}}$ \\
Severe disorder $\mathrm{n}(\%)$ & $6(9.68 \%)$ & $0(0)^{\mathrm{a}}$ & $5(8.19 \%)$ & $0(0)^{\mathrm{b}}$ \\
\hline
\end{tabular}

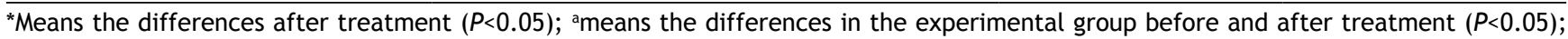
${ }^{b}$ means the differences in the control group before and after treatment $(P<0.05)$. 
TABLE 4: COMPARISON OF THE CURE RATE AFTER ESS

\begin{tabular}{lcccc}
\hline \multirow{2}{*}{ Group } & Cases & \multicolumn{3}{c}{ Cure rate } \\
\cline { 2 - 5 } & & $\mathbf{3 ~ m o}$ & $\mathbf{6 ~ m o}$ & $\mathbf{1 2}$ mo \\
\hline Control & 61 & $44(72.13)$ & $51(83.61)$ & $55(90.16)$ \\
Experimental & 62 & $51(82.25)$ & $54(87.10)$ & $58(95.16)$ \\
$\mathrm{t}$ & & 14.327 & 8.251 & 9.766 \\
$P$ & & $<0.05$ & $<0.05$ & $<0.05$ \\
\hline
\end{tabular}

period of $12 \mathrm{mo}$, indicating that the dexamethasone had a good effect on the postoperative recovery of ESS in a short period. CRS was extremely common in China, especially in the humid South China. With an increasing number every year, the CRS patients were becoming younger and younger, and this disease has the a characteristic of high recurrence rate, all of which make the CRS prevention and control more difficult and challenging ${ }^{[8]}$. There were many causes of CRS, including bacterial and fungal infections, nasal cilia transport disorders. The most important cause was the inflammatory infection in the complex of nasal sinus ostium and nasal meatus. The inflammation can promote the release of a large number of inflammatory factors, resulting in the mucosal edema, which stimulated the release of the inflammatory factors again to release more to form a nauseous cycle, and eventually leads to pathological changes in the anatomical structure of the complex of nasal sinus ostium and nasal meatus. And then CRS occurs ${ }^{[9-12]}$. It was accepted that the CRS can be treated only by the hormone medicines, and ESS can only be used for patients who have failed such treatment. The therapeutic schedule of hormone medicines will lead patients to enhance their compliance with hormone medicines, and easily lead to the relapse of the disease, so it is difficult to achieve the goal of radical cure. However, taking ESS treatment only after the failure of hormone medicines will not only delay the best treatment time, but also increase the cost of treatment and lead to the aggravation of the disease, even cancer. Therefore, at present, there is an increasing recognition on the clinical treatment of CRS by ESS combined with hormone medicines, but there is a lack of relevant research reports. The purpose of this study is to explore the clinical efficacy of ESS combined with the dexamethasone nasal spray in the treatment of CRS. With the advantages of visualization, accurate positioning, high accuracy, and slight trauma, ESS was widely used in the treatment of CRS. It can remove the pathological changes of the nasal cavity and sinuses accurately, improve and restore the functions of the nasal cavity and sinuses. In this study, ESS combined with dexamethasone was used to treat CRS and achieved good results. We followed up the patients in this study for one year, and found that during the follow-up period of 3, 6, and 12 mo after the treatment, the SNOT-20 scores and Lund-Kennedy scores in the experimental group were significantly lower, indicating that the treatment could improve the patients' condition significantly. The result is consistent with many previously reported results ${ }^{[12-14]}$. ESS was a very safe and convenient minimally invasive surgery, which could clear the disease symptoms and reconstruct the nasal structure effectively. It could play an important role in the treatment of CRS for reasons such as, ESS could improve ventilation in nasal cavity by cleaning and reconstructing the structure of the nasal cavity, so as to effectively improve the oxygen content of the nasal cavity and relieve symptoms of nasal obstruction. ESS could also clear the affected tissue at the junction of the sinuses and nasal cavity, so as to open airflow channels of the maxillary sinus, the anterior ethmoid sinus, and the frontal sinus. ESS could improve the absorption of drugs in the nasal cavity, and enhance the efficacy and duration of treatment ${ }^{[13]}$. It has been reported that the nasal cleaning after ESS was helpful to reduce the postoperative inflammatory response and improve the therapeutic effect ${ }^{[14]}$. Nasal cleaning facilitates in cleaning mucus and blood of the nasal cavity and repairing intranasal ciliary function ${ }^{[15]}$. Nasal cleaning can help to keep the nasal cavity moist and air circulation smooth. It can also reduce the secretory inflammation and edema symptoms in the nasal cavity, and promote the epithelial process of the nasal mucosa ${ }^{[16]}$. Dexamethasone was one of the most commonly used antiinflammatory glucocorticoid for CRS now. As dexamethasone is a glucocorticoid, patient must be monitored for hyperglycemia, especially in diabetic patients and for correcting this, dose of dexamethasone needed to be modified. On the other hand, dexamethasone also possesses convenient, efficient and lasting antiinflammatory effects, and directly inhibits the eosinophils in the nasal cavity, blocks the release of inflammatory substances like histamine and kallikrein, and protects the functions of the intraluminal mucosa and nasal cilia ${ }^{[17]}$. In addition, 
it can improve the stability of the lysosomal membrane and reduce the recurrence of CRS by regulating the migration process of some immune cells in the body, such as lymphocytes and macrophage ${ }^{[13]}$. This study was to evaluate the functional recovery of the nasal cavity by the postoperative olfactory tests. The results showed that after treatment for $12 \mathrm{mo}$, the olfactory function of the 2 groups was significantly improved, of which the olfactory function of $93.55 \%$ of patients in the experimental group was fully restored, and there were no patients with a mild degree or above in the experimental group, while only $73.77 \%$ of the patients in the control group had their olfactory functions fully restored. The difference between the 2 groups was significant, which indicated that dexamethasone could accelerate the postoperative recovery of patients significantly. At the same time, the results of this study also showed that after treatment, the cure rate of the 2 groups increased significantly in the follow-up period of 3,6 , and $12 \mathrm{mo}$, while the recurrence rate decreased significantly. Compared to the control group, the cure rate of the experimental group increased significantly at the first two follow-up periods. This indicated that ESS combined with dexamethasone has a good therapeutic outcome on CRS patients, which could improve their quality of life, and control the recurrence of the disease for a long time. This result is similar to the existing research reports ${ }^{[18-19]}$. In conclusion, ESS combined with dexamethasone is worth clinical promotion, for it can improve the clinical symptoms of CRS patients, improve their quality of life with better long-term efficacy.

\section{Declaration of interest:}

The authors report no conflicts of interest. The authors alone are responsible for the content and writing of the paper.

\section{REFERENCES}

1. Batko B, Stajszczyk M, Swierkot J, Urbanski K, Raciborski F, Jedrzejewski M, et al. Prevalence and clinical characteristics of rheumatoid arthritis in poland: A nationwide study. Arch Med Sci 2019;15:134-40.

2. Viecceli D, Garcia MP, Schneider L, Alegretti AP, Silva CK, Ribeiro AL, et al. Correlation between cellular expression of complement regulatory proteins with depletion and repopulation of b-lymphocytes in peripheral blood of patients with rheumatoid arthritis treated with rituximab. Rev Bras Reumatol 2017;57:385-91

3. Gomes RKS, Pires FA, Nobre MRC, Marchi MFS, Rickli JCK. Impact of rheumatoid arthritis in the public health system in santa catarina, brazil: A descriptive and temporal trend analysis from 1996 to 2009. Rev Bras Reumatol 2017;57:204-9.

4. Huang RY, Pan HD, Wu JQ, Zhou H, Li ZG, Qiu P, et al.
Comparison of combination therapy with methotrexate and sinomenine or leflunomide for active rheumatoid arthritis: A randomized controlled clinical trial. Phytomedicine 2019;57:403-10.

5. Hammaker D, Nygaard G, Kuhs A, Ai R, Boyle DL, Wang W, et al. Joint location-specific jak-stat signaling in rheumatoid arthritis fibroblast-like synoviocytes. ACR open rheumatology 2019;1:640-8.

6. Zhang Q, Liu J, Zhang M, Wei S, Li R, Gao Y, et al. Apoptosis induction of fibroblast-like synoviocytes is an important molecular-mechanism for herbal medicine along with its active components in treating rheumatoid arthritis. Biomolecules 2019;9(12):795.

7. Bi X, Guo XH, Mo BY, Wang ML, Luo XQ, Chen YX, et al. Lncrna picsar promotes cell proliferation, migration and invasion of fibroblast-like synoviocytes by sponging mirna4701-5p in rheumatoid arthritis. EBioMedicine 2019;50:40820.

8. Allmer J, Yousef M. Computational mirnomics. J Integr Bioinform 2016;13:1-2

9. Wang Y, Zeng J, Pan J, Geng X, Liu Y, Wu J, et al. Microrna$200 \mathrm{c}$ is involved in proliferation of gastric cancer by directly repressing p27(kip1). Biochem Biophys Rep 2016;8:227-33.

10. Wang Y, Feng T, Duan S, Shi Y, Li S, Zhang X, et al. Mir155 promotes fibroblast-like synoviocyte proliferation and inflammatory cytokine secretion in rheumatoid arthritis by targeting foxo3a. Exp Ther Med 2020;19:1288-96.

11. Dinesh P, Kalaiselvan S, Sujitha S, Rasool M. Mir-145-5p mitigates dysregulated wnt1/beta-catenin signaling pathway in rheumatoid arthritis. Int Immunopharmacol 2020;82:106328.

12. Li B, Zhou C, Yi L, Xu L, Xu M. Effect and molecular mechanism of mtor inhibitor rapamycin on temozolomideinduced autophagic death of u251 glioma cells. Oncol Lett 2018;15:2477-2484

13. Yu M, Gong ZP, Ma ZL, Yao ZF, Liu C, Wang J, et al. Crkl promotes proliferation, migration, invasion of laryngeal squamous cell carcinoma. Eur Rev Med Pharmacol Sci 2020;24:5473-80.

14. Palit P, Ghosh R, Tolani P, Tarafdar A, Chitikineni A, Bajaj $\mathrm{P}$, et al. Molecular and physiological alterations under elevated co2 concentrations in chickpea. Plant Cell Physiol. 2020;61(8):1449-63.

15. Li Y, Wang LM, Xu JZ, Tian K, Gu CX, Li ZF. Gastrodia elata attenuates inflammatory response by inhibiting the nf-kappab pathway in rheumatoid arthritis fibroblast-like synoviocytes. Biomed Pharmacother2017;85:177-81

16. Lebre MC, Vieira PL, Tang MW, Aarrass S, Helder B, Newsom-Davis T, et al. Synovial il-21/tnf-producing cd4(+) $\mathrm{t}$ cells induce joint destruction in rheumatoid arthritis by inducing matrix metalloproteinase production by fibroblastlike synoviocytes. J leukocyte biol 2017;101:775-83.

17. Guo Q, Zhang S, Huang J, Liu K. Alogliptin inhibits il-1betainduced inflammatory response in fibroblast-like synoviocytes. Int immunopharmacol 2020;83:106372.

18. Wei Q, Lv F, Zhang H, Wang X, Geng Q, Zhang X, et al. Microrna-101-3p inhibits fibroblast-like synoviocyte proliferation and inflammation in rheumatoid arthritis by targeting ptgs2. Biosci Rep 2020;40(1):BSR20191136.

19. Cai Y, Jiang C, Zhu J, Xu K, Ren X, Xu L, et al. Mir-449a inhibits cell proliferation, migration, and inflammation by regulating high-mobility group box protein 1 and forms a mutual inhibition loop with yin yang 1 in rheumatoid arthritis 
fibroblast-like synoviocytes. Arthritis Res Ther 2019;21:134.

20. Wang J, Zhao Q. Betulinic acid inhibits cell proliferation, migration, and inflammatory response in rheumatoid arthritis fibroblast-like synoviocytes. J Cell Biochem 2018.

21. Ma JD, Jing J, Wang JW, Yan T, Li QH, Mo YQ, et al. A novel function of artesunate on inhibiting migration and invasion of fibroblast-like synoviocytes from rheumatoid arthritis patients. Arthritis Res Ther 2019;21:153.

22. Bustamante MF, Oliveira PG, Garcia-Carbonell R, Croft AP, Smith JM, Serrano RL, et al. Hexokinase 2 as a novel selective metabolic target for rheumatoid arthritis. Ann Rheum Dis 2018;77:1636-43.

23. Huang Z, Xing S, Liu M, Deng W, Wang Y, Huang Z, et al. Mir$26 \mathrm{a}-5 \mathrm{p}$ enhances cells proliferation, invasion, and apoptosis resistance of fibroblast-like synoviocytes in rheumatoid arthritis by regulating pten/pi3k/akt pathway. Biosci Rep 2019;39(7):BSR20182192.
24. Lenis YY, Wang X, Tang W, Wu G, Bazer FW. Effects of agmatine on secretion of interferon tau and catecholamines and expression of genes related to production of polyamines by ovine trophectoderm cells. Amino Acids 2016;48:2389-99.

25. Zhao QG, Lu BS, Huang PT. Functions of fancl in primordial germ cell formation and fanconi anemia. Yi Chuan Xue Bao2005;32:993-1000.

This is an open access article distributed under the terms of the Creative Commons Attribution-NonCommercial-ShareAlike 3.0 License, which allows others to remix, tweak, and build upon the work non-commercially, as long as the author is credited and the new creations are licensed under the identical terms

This article was originally published in a special issue, "Biomedical Research in Healthcare Setting" Indian J Pharm Sci 2020:82(3)Spl issue5;124-129 